\title{
Effect of tidal stream power generation on the region-wide circulation in a shallow sea
}

\author{
G. I. Shapiro \\ University of Plymouth, Drake Circus, Plymouth, PL4 8AA, UK \\ Received: 1 October 2010 - Published in Ocean Sci. Discuss.: 21 October 2010 \\ Revised: 11 February 2011 - Accepted: 15 February 2011 - Published: 24 February 2011
}

\begin{abstract}
This paper quantifies the backward effect on the ocean currents caused by a tidal stream farm located in the open shallow sea. Recent studies in channels with 1-D models have indicated that the power potential is not given purely by the flux of kinetic energy, as has been commonly assumed. In this study, a 3-D ocean circulation model is used to estimate (i) practically extractable energy resource at different levels of rated generation capacity of the farm, (ii) changes in the strength of currents due to energy extraction, and (iii) alterations in the pattern of residual currents and the pathways of passive tracers. As well as tidal streams, the model also takes into account the wind-driven and density-driven ocean currents. Numerical modelling has been carried out for a hypothetical tidal farm located in the Celtic Sea north of Cornwall, an area known for its high level of tidal energy. Modelling results clearly indicate that the extracted power does not grow linearly with the increase in the rated capacity of the farm. For the case study covered in this paper, a 100-fold increase in the rated generation capacity of the farm results in only 7-fold increase in extracted power. In the case of a high power farm, kinetic energy of currents is altered significantly as far as $10-20 \mathrm{~km}$ away from the farm. At high levels of extracted energy the currents tend to avoid flowing through the farm, an effect which is not captured with 1-D models. Residual currents are altered as far as a hundred kilometres away. The magnitude of changes in the dispersion of tracers is highly sensitive to the location. Some of the passive drifters analysed in this study experience significant variations in the end-to-start distance due to energy extraction ranging from $13 \%$ to $238 \%$ while others are practically unaffected. This study shows that both energy extraction
\end{abstract}

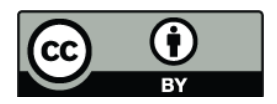

Correspondence to: G. I. Shapiro (gshapiro@plymouth.ac.uk) estimates and effects on region wide circulation depend on a complex combination of factors, and the specific figures given in the paper should be generally considered as first estimates.

\section{Introduction}

Ocean tides are driven by the Earth's rotation in combination with gravitational forces from the Sun and the Moon. As such, tidal energy is almost inexhaustible despite the gradual reduction of kinetic energy of the Earth's rotation due to the dissipation of tidal currents via friction and turbulence. This process is very slow, during the last 620 million years the Earth has lost only $17 \%$ of its rotational energy (Williams, 2000). However, similar to other energy resources, the amount of practically extractable energy is limited. The Energy White Paper (DTI, 2003) states that in order to meet its climate change targets, the UK would need to produce by 2050 at least $30 \%$ to $40 \%$ of its electricity generation from renewables. Some of the large renewable power stations of the future will be offshore marine plants, including wave, tidal and wind farms.

Total rate of dissipation (and hence the rate of production) of tidal energy in the World ocean is known from astronomy to be 3.7 TW (Munk and Wunsch, 1998). The tidal dissipation in the Earth's shelf seas is on average about 2.5 TW (Egbert and Ray, 2000) or $21900 \mathrm{TWh} \mathrm{yr}^{-1}$, which is comparable with the world consumption of electricity of $19771 \mathrm{TWh} \mathrm{yr}^{-1}$ in 2007, see (IEA, 2009). The usable World ocean resource of tidal energy is estimated at $800 \mathrm{TWh} \mathrm{yr}^{-1}$ (Soerensen and Weinstein, 2008). Tidal power is considered to be an important source of renewable energy for the UK and those European countries which are

Published by Copernicus Publications on behalf of the European Geosciences Union. 
exposed to high levels of tidal energy. Despite its relatively high cost, the generation of electricity from tidal energy may be a potential stable source of renewable energy for the future.

Tidal energy has two components, the first is the potential energy due to sea level variations, and the second comes from the kinetic energy of the tidal streams. It is the potential energy of the tides which is commonly used for electricity generation. As of August 2010, there are 6 commercially operational tidal power plants: La Rance, France (240 MW capacity, built in 1966); Kislaya Guba Bay, Russia (1.7 MW, 1968); Annapolis, Canada (20 MW, 1985); Strangford Lough, Northern Ireland (1.2 MW, 2008); Uldolmok, South Korea (1.0 MW, 2009), Jiangxia, China (3.2 MW, 1980) - see (List of largest power stations in the world, 2010) for more details. Four out of six plants use barrages or dams to create a difference in water level, while the Strangford Lough device, which uses twin $16 \mathrm{~m}$ diameter rotors (Marine Current turbines, 2010) and the Uldolmok device (Gorlov, 2001) are the only commercial scale tidal stream power generators.

The engineering aspects and potential environmental impacts of tidal barrages, which use the potential energy of the tide, have been given a thorough consideration; (see e.g. Xia et al., 2010; DE, 1989; SDC, 2007). By comparison, our knowledge of how tidal stream devices interact with the marine environment is limited. A tidal stream device is typically a submerged underwater turbine used for harnessing energy from ocean currents. A group of such turbines distributed in a site is called a tidal stream farm which is similar to a wind farm (Li et al., 2009). Recent research has shown that tidal stream devices located in channels can significantly decrease the velocity of the water. The total energy available for harnessing is then reduced by the presence of the turbines, so that the commonly used formula for the maximum available power does not give a true estimate of the practically extractable energy (Garrett and Cummins, 2008; Bryden and Couch, 2006; Mueller and Wallace, 2008). On the oceanic shelf, studies of distortion of ocean currents by tidal stream devices and the environmental impact of such alterations are extremely sparse (Neill et al., 2009). Little is known about region-wide impacts of ocean energy extraction, and how far away from the actual device they can be seen. These issues have still to be explored and have motivated the present study.

This paper uses a 3-D ocean circulation model (i) to assess alterations to the circulation pattern in the shelf sea by a hypothetical tidal stream energy farm, (ii) to estimate how these alterations impact on the availability of extractable ocean energy, and (iii) to explore how the process of energy extraction changes both instantaneous and residual (tidally averaged) water flow in the wider region up to a hundred kilometres away from the farm. It has to be noted that despite being named "tidal energy converters" the tidal stream devices located on the shelf extract energy from the total incoming current which is composed of wind driven and density driven currents as well as the tidal stream. This notion is particularly important in the assessment of the alteration to residual currents and the pathways of dispersion of pollutants on the shelf.

\section{Shelf sea dynamics and harnessing of ocean kinetic energy}

It is common sense that if kinetic energy is extracted from the sea then current speeds should be affected as kinetic energy is directly related to the velocity of the current. The kinetic energy, KE, of a water mass is its ability to do work by virtue of its motion. The kinetic energy per unit volume of a fluid parcel moving with velocity $u$ is

$\mathrm{KE}=\frac{1}{2} \rho u^{2}$

and the energy flux through a "window" of unit area perpendicular to the fluid flow (i.e. vertical in case of ocean currents) is

$P=\mathrm{KE} \times u=\frac{1}{2} \rho u^{3}$

This equation is widely used to assess the "theoretically available" tidal energy resource (e.g. Soerensen and Weinstein 2008; Walkington and Burrows, 2009; Carballo et al., 2009) without considering the reduction of the incoming flow rate due to energy extraction. However such an approach has been criticized by Garrett and Cummins (2008).

The upper limit of extractable tidal stream energy (and generally kinetic energy of currents of any origin) given by Eq. (2) assumes that while passing through the "window" the water flow is subjected to such extreme friction that the current collapses and its speed falls to zero after the passage. Through the law of mass conservation there would be an obvious effect on the speed of the incoming current. As a result, the true "theoretically available" power may be significantly lower than that given by Eq. (2) under the assumption that the speed of the incoming flow was not disturbed. One approach to take this feedback into account is to scale down the value given by Eq. (2) by multiplying its right hand side by a prescribed "efficiency coefficient" (Carballo et al., 2009). Another approach was employed in the modelling study of the near-field flow around the tidal turbine by Walkington and Burrows (2009). The reduction of vertically integrated flow speed was obtained by increasing the bottom drag coefficient within the energy farm .The drag coefficient was then determined from the prescribed rated power of the tidal device at a given inflow velocity.

In their study of flow speed reduction due to energy extraction in a channel, Garrett and Cummins (2008) assumed that turbines are deployed in a uniform or a partial fence, or fences, occupying a line across the channel. The authors use a quasi 1-D approach when all parameters are integrated over 
a transect perpendicular to the current flow. The energy loss per unit time associated with the presence of the turbines is then given by

$P=\rho Q \int_{0}^{L} F_{\text {turb }} d x$

where $\rho$ is the density of water, $Q$ is the flux of water through the channel, $F_{\text {turb }}$ is the cross-sectional average of retarding frictional forces associated with the presence of turbines, $x$ is the distance along the channel, and $L$ is the total length of the channel.

The maximum available power, $P_{\max }$, is given by the equation

$P_{\max }=0.38 \rho Q_{0} g \zeta_{0}$

where $Q_{0}$ is the natural flux (without turbines), and $\zeta_{0}$ is the difference in sea level elevation at the entrance and exit of the channel. This formula gives a helpful estimate of energy extraction in a channel; however, the numerical factor may vary slightly owing to acceleration and the formula is not applicable in the open ocean setting, where lateral deviations of the current may play a role.

In this paper we examine how a tidal energy farm placed on the open shelf modifies ocean currents using a different approach, namely a 3-D ocean circulation model. This approach allows assessing the rate of energy extraction by a geometrically compact (i.e. not of a barrage/fence type) energy farm and its impact on the circulation around the farm. The currents in the shelf sea are subject to various forces: water column and bottom friction, wind stress, pressure gradients due to temperature and salinity variations, all of which contribute to the formation of complex 3-D flow patterns even in a tidally dominated sea. The use of a 3-D numerical model allows these patterns to be quantified.

The horizontal momentum equation for ocean currents in the presence of tidal stream devices can be written as

$\rho \frac{\partial u}{\partial t}=\mathrm{NL}+F$

where $\rho$ is the density of fluid, NL represents the standard terms of the nonlinear momentum equation including advection, the Coriolis force, pressure gradient force and natural friction (Pedlosky, 1990), and $F$ is the flow retarding force per unit volume due to the presence of energy devices.

According to Newton's third law of motion, the retarding, or drag, force exerted on the flow by a turbine is equal and opposite to the thrust, $F_{\mathrm{T}}$, exerted by the flow on the turbine. The thrust is related to the speed of the flow via the following equation, see e.g. (Hansen, 2008):

$F_{\mathrm{T}}=\frac{1}{2} C_{\mathrm{T}} \rho u^{2} A ;$

where $C_{\mathrm{T}}$ is the thrust coefficient and $A$ is the cross-sectional area of the flow passing through the turbine.

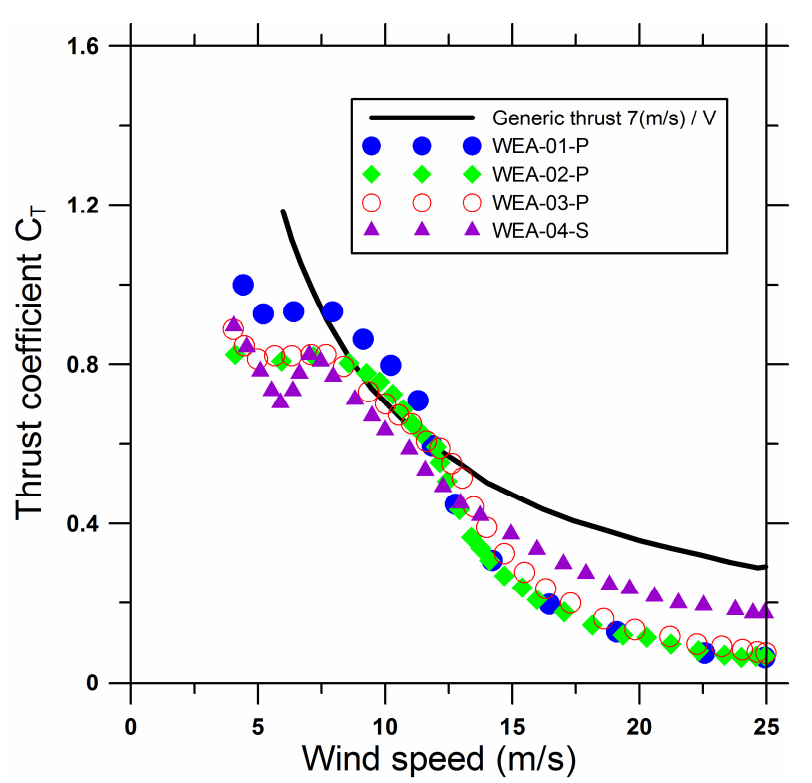

Fig. 1. The thrust coefficient, $C_{\mathrm{T}}$, as a function of wind speed, $u$, for a number of commercially available wind turbines (WEA01...WEA-04) together with approximating curve given by Eq. (7). Based on data from Frohboese and Schmuck (2010).

For a turbine, the thrust coefficient, $C_{\mathrm{T}}$, is not a constant but is highly dependent on the speed of the incoming flow, see e.g. Leishman (2000). Strong dependence of $C_{\mathrm{T}}$ on the current speed makes the dynamics of flow through turbines very different from the turbulent flow around static objects such as a disk or a plate where a similar quantity, the drag coefficient, $C_{\mathrm{d}}$, could be reasonably assumed constant within a wide range of Reynolds numbers. The physical reason for such a difference is that in case of a turbine the retarding force is mainly caused by the transfer of momentum and energy from the flow to the rotating turbine blades. On the contrary, in the turbulent flow around a static object the frictional drag is mainly caused due to generation of turbulence by the object. As engineers aim to reduce unwanted mechanical energy losses in the turbines, the bulk of the flow energy goes into rotation of the turbine blades.

Experimental evidence shows (Frandsen, 2007, Eq. 3.12) that within the working range the thrust coefficient $C_{\mathrm{T}}$ can be approximated as inversely proportional to the flow speed, an approximation that is supposed to fit for most of the modern wind turbines (Frohboese and Schmuck, 2010):

$C_{\mathrm{T}}=\frac{\text { Const }}{u}$

Figure 1 shows results of measurements obtained for a number of commercially available wind turbines as well as the curve computed using Eq. (7) and Const $=7 \mathrm{~m} \mathrm{~s}^{-1}$ (Frohboese and Schmuck, 2010). A combination of 
Eqs. (7) and (6) results in a linear law of "friction" exerted by a rotating turbine

$F=-\frac{1}{2} \alpha \rho u$

rather than a "quadratic law" commonly used to describe turbulent friction around static objects. The vector notation is used here to indicate the direction of the retarding force, and a new constant $\alpha=$ Const $\times A$ is introduced for brevity.

Mechanical power transferred from the flow to the turbine blades is given by the work done by the retarding force per unit time, e.g. (Hansen, 2008); using Eq. (6) one can get

$P=\rho F u=\frac{1}{2} C_{\mathrm{T}} \rho u^{3} A$

By multiplying Eq. (8) by $u$ one can show that the linear law for the retarding force results in a quadratic law for the loss of kinetic energy of the flow

$P=\rho F u=\frac{d \mathrm{KE}}{d t}=\frac{1}{2} \alpha \rho u^{2}=-\alpha \mathrm{KE}$

Equation (10) shows that the rate of energy extraction per unit volume, $P$, is proportional to $u^{2}$, and hence the power efficiency of a turbine relative to the flux of arriving kinetic energy, which is proportional to $u^{3}$, see Eq. (2), decreases with increasing flow. This conclusion is in agreement with measurements of efficiency of wind turbines with horizontal axes (Stiesdal et al., 1999). Equation (10) has a simple physical interpretation: the loss of energy, $P$, is proportional to the actual (i.e. modified by the energy farm) kinetic energy of the flow, KE.

The linear dependence of the retarding force on the flow speed is similar to the Rayleigh friction scheme, which is often used in meteorology to parameterize the friction within the air flow, e.g. (Bjorn et al., 2002), with $\alpha$ being equivalent to the Rayleigh friction coefficient. The form of Eq. (10) integrated over the volume of a numerical gridbox is used in this paper for quantifying energy extracted from ocean currents.

\section{The model}

The numerical model used in this study is a finite difference 3-D numerical ocean circulation model POLCOMS developed in the Proudman Oceanographic Laboratory (Holt and James, 2001). It has been thoroughly validated for UK waters (Holt et al., 2005; Siddorn et al., 2007; Lewis et al., 2006) and has been used in many research projects and operationally by the MetOffice (without energy extraction terms). The model uses an Arakawa B-grid in the horizontal and terrain following s-coordinate in the vertical. For this study the model was set up for the eastern Celtic Sea including the Bristol Channel (Shapiro et al., 2010); the area is known for its high tidal energy level (Huntley, 1980), see Fig. 2. The bottom topography was acquired from the ETOPO2

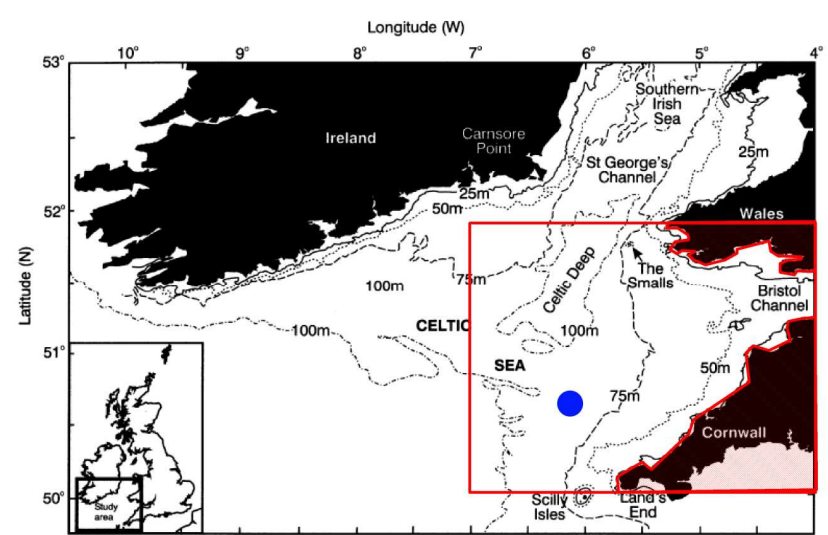

Fig. 2. Bathymetric map of the Celtic Sea showing the study area (red rectangle) and location of the tidal farm (blue spot).

global bathymetry data base (ETOPO2, 2006), initial boundary conditions for temperature and salinity were taken from the climatology presented in the World Ocean Atlas (Boyer et al., 2005), meteorological forcing was obtained from the NCEP reanalysis-2 dataset. Tidal boundary conditions for 15 tidal constituents were obtained from larger scale POLCOMS modelling runs carried out in the Proudman Laboratory (S. Wakelin, personal communication, 2009). For this study, the model was run with 30 vertical levels, and $2 \mathrm{~km}$ horizontal resolution. In order to exclude effects of wetting and drying, which are not relevant to the present study, numerical calculations were carried out only in the areas deeper than $10 \mathrm{~m}$. The model code was modified to include the additional frictional forces due to energy extraction as detailed in the previous section.

As development of "tidal stream" energy generators is in its infancy, little is known about the details of the future industrial scale energy farm. A hypothetical "tidal stream" energy farm incorporated into the numerical model has a circular shape in the horizontal plane, and the energy converters were assumed to be able to capture energy throughout the whole water column, from the bottom to the surface. As we do not know the exact vertical structure of future tidal farms, the uniform distribution of energy extraction in the vertical seems to be a logical first step. Such an approach is effectively used in 2-D (vertically integrated) numerical models, e.g. Neill et al. (2009). As more details of the energy devices become available, the 3-D approach would allow us to assess the effects of non-homogenous power extraction. In contrast to the existing commercially operational tidal plants, which are installed in dams or barrages, this hypothetical farm contains an array of underwater turbines distributed over a compact area of the shelf sea.

The values of the Rayleigh "friction" coefficients $(\alpha)$ averaged over each numerical grid box represent the relative intensity of the kinetic energy loss due to turbines. As the 

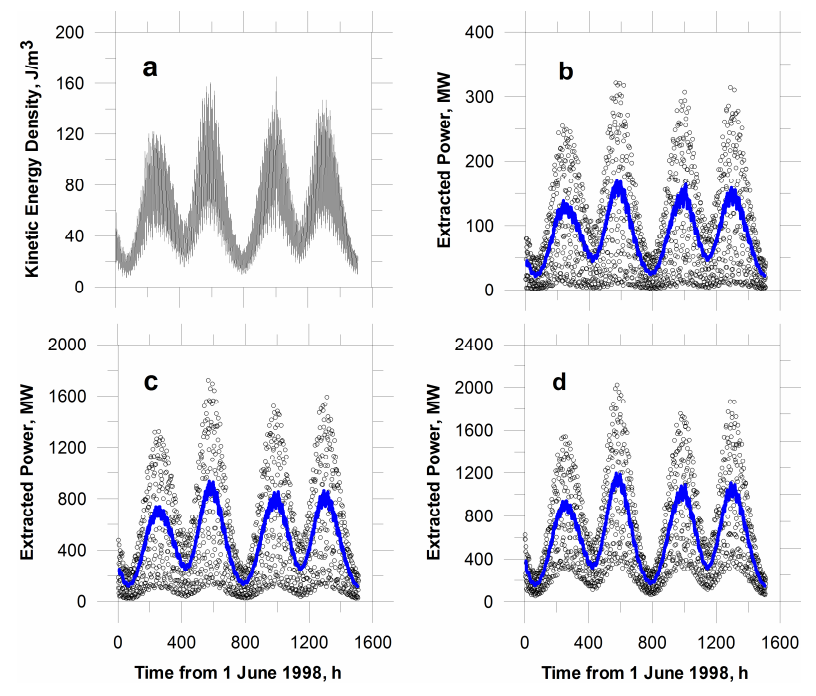

Fig. 3. Time series of kinetic energy per unit volume averaged over the study area (a) and power loss from ocean currents due to tidal energy farm at different rates of energy extraction: (b) low, (c) medium, and (d) high rated capacity farm. Open circles show values of power extraction spatially integrated over the whole model domain, solid curves show a running mean with a $12 \mathrm{~h}$ averaging window.

model is concerned with the cumulative action of the energy devices, the box-averaged values of $\alpha$ represent both the number of turbines inside the box and the efficiency of devices; larger values correspond to greater energy losses.

The eastern Celtic Sea is known for its highly stratified structure and the sharp density fronts present during the summer months. These fronts contribute to the formation of density-driven currents which, as well as wind-driven currents, can influence the transport of water mass over periods longer than the tidal cycle. As the numerical model calculates not only the tidal streams but also the total ocean current the results are specific to the time period chosen for modelling, not just to the phases of the tidal cycle.

\section{Results and discussion}

For this study, a period of relatively calm weather was chosen, namely between the 1st June and 2nd August 1998, which covered 4 spring-neap cycles. Numerical runs were performed mainly for a farm of a circular shape. For comparison some runs were made for an elongated farm. The diameter of the circular farm was $12 \mathrm{~km}$. The values of $\alpha$ were set constant within the farm and zero outside it, and then a Gaussian smoothing was applied to avoid numerical instability. The runs were carried out for the baseline situation (no farm present) and with 3 different values of the rated power of the farm represented by different values of the maximum value of $\alpha_{0}$ within the farm:

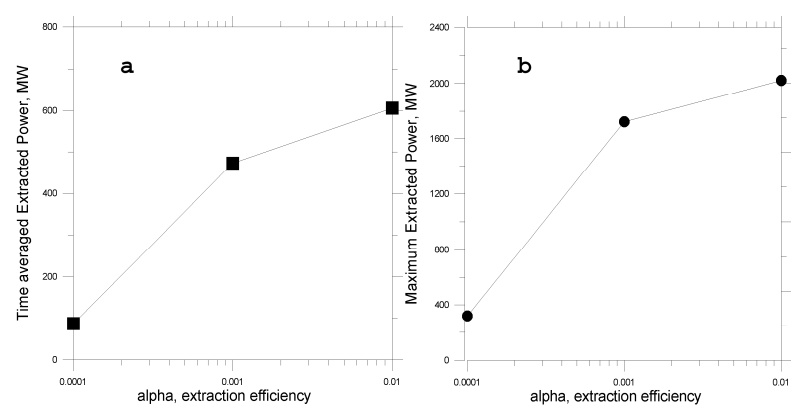

Fig. 4. Mean and maximum extracted energy by the tidal energy conversion farm as a function of the rated power capacity of the farm represented by the Rayleigh coefficient $\alpha$.

(L) $\alpha_{0 \mathrm{~L}}=10^{-4}, s^{-1}$

(M) $\alpha_{0 \mathrm{M}}=10 \times \alpha_{0 L}$;

(H) $\alpha_{0 \mathrm{H}}=100 \times \alpha_{0 L}$.

Comparison of these runs allows us to examine the relative efficiency of power extraction and the environmental impact of the energy generation of the farm with differing rated power capacity. The values of the Rayleigh coefficient were selected to represent a wide range of the mean extracted power- from $87 \mathrm{MW}$ to $606 \mathrm{MW}$, see below. It should be noted that the increase in the Rayleigh coefficient $\alpha$ is proportional to the increase in the rated power of the farm, either due to a greater density of turbines within the farm or the increase in the rated power of individual devices. In other words, the cases (L), (M) and (H) correspond respectively to a relatively low energy farm, medium range (having a capacity rated 10 times greater) and a high energy farm with a capacity rated 100 times greater. The terms "low", "medium" and "large" are used in a relative sense; we shall see that even a "low" energy farm can extract as much as $87 \mathrm{MW}$ on average.

In the Celtic Sea the currents are dominated by the tidal streams, so it is not a surprise that the values of kinetic energy for the dates corresponding to the same phase in the springneap cycle are fairly similar. The time series of the kinetic energy per unit volume averaged over the depth and area of the modelling domain is shown in Fig. 3a for the baseline case (i.e. without the energy farm). Temporal variations of kinetic energy when the farm is present have a similar shape.

The time series of the power loss by the currents due to the energy farm are shown in Fig. 3b-d. The temporal patterns for all 3 cases are very similar and follow the spring-neap cycle of the ocean current kinetic energy in the region. However there is a significant difference in the magnitude of the extracted power. If the electrical efficiency of the turbines stays the same (or nearly the same) in all cases, i.e. if the amount of electrical power generated is proportional to the amount of energy taken from the sea by the turbines, this would mean significant variations in the electric power output from the farm. Figure 4 shows the time averaged values 

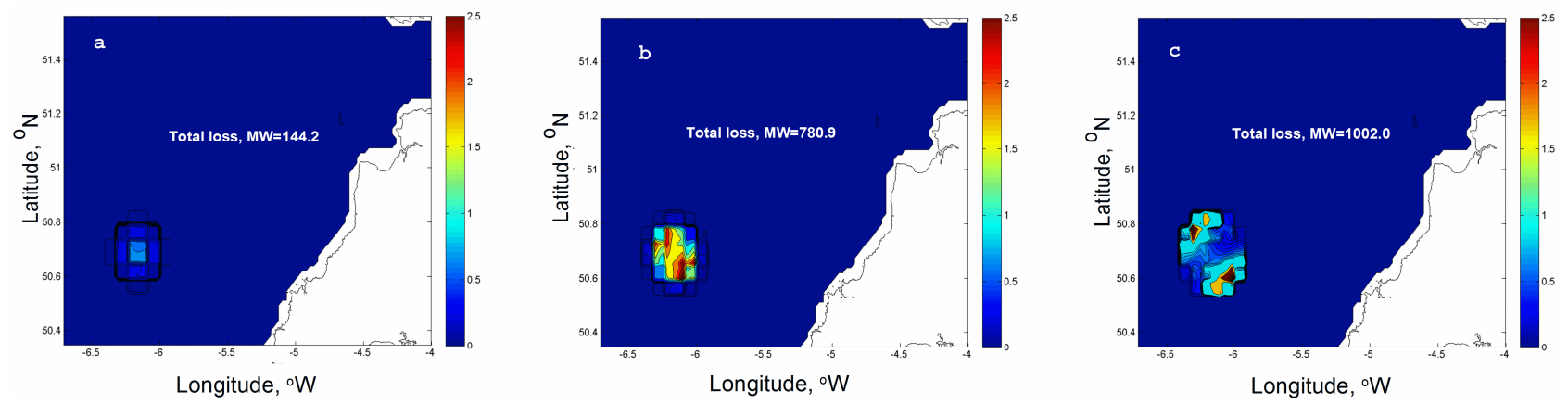

Fig. 5. Spatial distribution of the mean extracted power in $\mathrm{W} \mathrm{m}^{-2}$ for the 3 versions of the tidal energy farm: (a) low, (b) medium and (c) high rated power capacity. Averaging is performed over a 50-hour period during the spring tides. The total (area integrated) value of extracted power averaged over the same period is also shown.

of the extracted power for the 3 cases, the averaging period being from 1st June to 8th July, as well as maximum values of extracted energy over the same period.

Due to the backward effect of the energy extraction on the ocean currents, the increase in the rated power capacity of the farm provides less than linearly proportional increase in the actual extracted power. The mean extracted power from the lowest rated farm " $\mathrm{L}$ " is $87 \mathrm{MW}$, a 10 -fold increase in the rated power of the farm (case "M") gives only a 5.5-fold increase in the harnessed energy at $473 \mathrm{MW}$, and a 100-fold increase of effort (case " $\mathrm{H}$ ") provides only $606 \mathrm{MW}$ of mean extracted power, a 7-fold increase in extracted energy as compared to case " $\mathrm{L}$ ". The maximum (instantaneous) power levels for the three versions of the farm are $0.3 \mathrm{GW}, 1.7 \mathrm{GW}$ and $2 \mathrm{GW}$ respectively. If the extracted power had been proportional to the rated capacity of the farm, then the high energy farm would have harnessed on average a massive $8.7 \mathrm{GW}$ of tidal energy with a peak value of $30 \mathrm{GW}$. These hypothetical values are unrealistic - the total

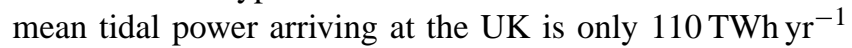
or $12.5 \mathrm{GW}$ (Black and Veatch, 2005). Fig. 4. clearly shows that when the level of power generation is high, then the efficiency of energy generation decreases. Power generation grows slower than the effort (e.g. density of turbines in the farm, or rated power of individual turbines) as the loss of kinetic energy reduces the amount of energy available for further utilisation. Fig. 5 shows that in the medium and high rated energy farms most of energy generation occurs at the periphery of the farm; the turbines in the centre generate energy from already reduced water flow.

A conceptually similar effect predicting the reduction of the maximum extractable power due to the influence of turbines on the current in a channel was reported earlier (Garrett and Cummins, 2008; Bryden and Couch, 2006). The difference here is that in the open sea the effect is much stronger: in a channel the maximum extractable power was reduced only by a factor of 3 (Garrett and Cummins, 2008), in the open sea the decrease can be as high as 14-fold (see case
" $\mathrm{H}$ "). The main reason is that in the open sea the currents can avoid flowing through a localised energy farm. In a channel, the slow-down of the flow in front of the turbine causes an increase in the sea level forming a pressure gradient that partially mitigates the loss of flow. The recovery of kinetic energy due to increases in the potential energy has a much weaker effect in the open sea. Hence, the effect of reduction of maximum extractable power is stronger in the 3-D case considered here as compared to assessments done for 1-D geometry (Carballo et al., 2009; Walkington and Burrows, 2009). Walkington and Burrows (2009) also carried out 2-D modelling and noted a similar effect of current going around the farm to avoid the "blockage" so that power extraction is reduced.

The spatial distribution of the extracted tidal power is shown in Fig. 5. In order to filter out the oscillation of the extracted power within a tidal cycle, the value of $P$ shown in Fig. 5 is averaged over $50 \mathrm{~h}$ (i.e. slightly over 4 tidal cycles, there is no exact periodicity due to wind-driven and density-driven currents), corresponding to the middle of the 3rd spring tide period in Fig. 3a. In the case of the low power farm " $L$ ", the extracted power is maximum in the central part of the farm, i.e. at the location with the highest rated capacity of energy conversion per unit area. In case of the high power farm " $\mathrm{H}$ ", the central parts of the farm produce less energy than some of the peripheral locations (a kind of a sheltering effect). The reduction in the level of energy extraction in the central parts is due to the slowing down of ocean currents by the process of energy extraction at the periphery of the farm.

The negative feedback of the tidal energy farm on the strength of the currents within and around the farm is seen from the charts of depth-integrated kinetic energy shown in Fig. 6. The low values of depth-integrated kinetic energy in the coastal regions are mainly due to the shallow water depth and hence smaller volumes of water involved in the movement.

The presence of the low energy farm "L" (Fig. 6b) decreases the highest levels of the kinetic energy of the currents 

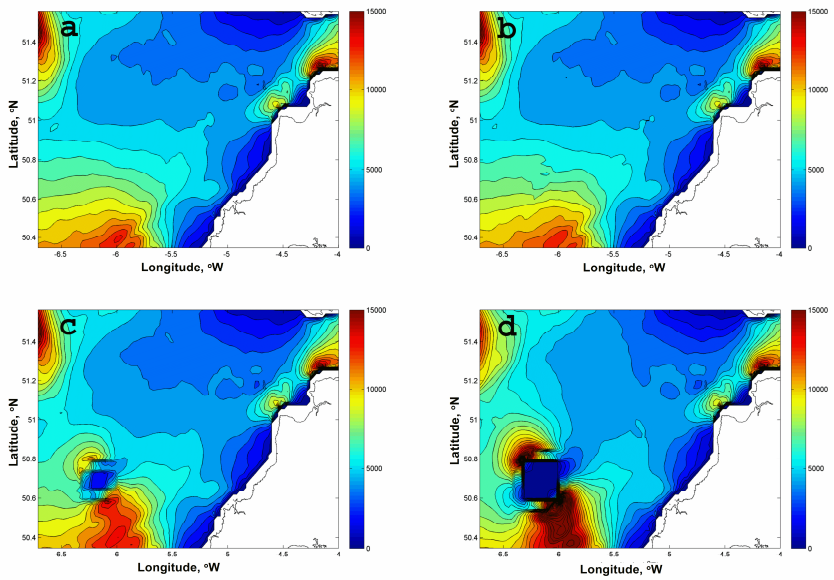

Fig. 6. Spatial distribution of kinetic energy per unit area $\left(\mathrm{J} \mathrm{m}^{-2}\right)$ integrated over depth from bottom to the surface and averaged over $50 \mathrm{~h}$. Time averaging is centred around the spring high water corresponding to the 2 nd spring period in Fig. 3. The charts represent 4 cases: (a) the baseline case (no energy extraction) (b) low, (c) medium, and (d) high rated power capacity farm.

in the area but does not change the spatial pattern, so that the location of the farm is not evident from the chart of ocean energy. A 10-fold increase in the rated power (case "M") results in a more localised loss of kinetic energy within and in the close vicinity of the farm. The low levels of kinetic energy in the coastal areas remain practically unchanged. The high energy farm " $\mathrm{H}$ " significantly influences the levels of kinetic energy not only within the farm but also a few tens of kilometres away. In areas neighbouring the farm to the North-east and South-west the levels of KE are much lower than in the baseline case. By contrast, the energy levels to the North-west and South-east have increased. With such a great capacity to absorb energy from the current, farm " $\mathrm{H}$ " acts as an obstacle, nearly as an island, and deflects the currents so that they concentrate in narrow areas around the "island".

The effect of the farm on the residual (tidally averaged) currents is seen from the trajectories of Lagrangean floats deployed in the model at various locations and drogued at $22.5 \mathrm{~m}$ depth, see Fig. 7. This figure shows trajectories of the drifters for the period 1st June to 8th July. Drifter \#1 is deployed in the coastal waters SE of the farm and travels first to the NE and then heads for the Severn Estuary. It does not change its trajectory even in the presence of the high power farm. Drifter \#7 is deployed in the centre of the farm. In the baseline case it slowly moves to the south. In the presence of the farm " $L$ " it circulates around the farm. In the presence of more powerful farms "M" and " $\mathrm{H}$ ", this drifter is trapped within the farm; this effect is particularly prominent in the case of the high energy farm: the trajectory is so compact that it is hidden behind the label "7" in Fig. 7d. Drifter \#8 is deployed in between drifters \#1 and \#7. The presence of the
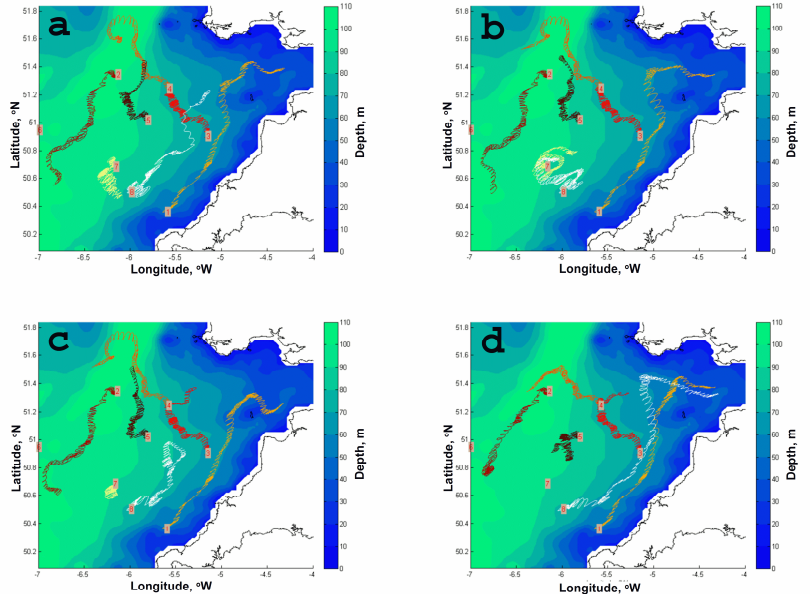

Fig. 7. Simulated trajectories of Lagrangean drifters drogued at $22.5 \mathrm{~m}$ depth. (a) baseline case, (b) low, (c) medium, and (d) high rated power capacity farm for the period from 00:00 $\mathrm{h} 1$ June to 13:00 h 08 July 1998. The centre of the farm coincides with the launch site of drifter \#7.

Table 1. Travel distance by Lagrangean floats over 30 days.

\begin{tabular}{|c|c|c|c|c|c|c|c|}
\hline $\begin{array}{l}\text { Drifter \# } \\
\text { Travel distance in the } \\
\text { baseline case, } \mathrm{km}\end{array}$ & 137 & 82 & 44 & 62 & 25 & 18 & 55 \\
\hline \multicolumn{8}{|c|}{ Distance travelled as percentage of the baseline case, $\%$} \\
\hline & 95 & 92 & 10 & 97 & 123 & 109 & \\
\hline Farm "M" & 94 & 100 & 108 & 104 & 132 & 24 & 113 \\
\hline Farm "H" & 99 & 97 & 115 & 74 & 51 & 13 & 238 \\
\hline
\end{tabular}

low power farm "L" causes its trajectory to be confined by the area around the farm. The impact of the medium power farm " $\mathrm{M}$ " causes its advection towards the Northeast, whilst in the presence of the high power farm " $\mathrm{H}$ ", this drifter accelerates and follows drifter \#1 on its way to the Severn Estuary. Other drifters modify their trajectories in a variety of ways as shown in Fig. 7.

Changes in the travelling distance, i.e. the distance between the start and the end points for 7 out of 8 modelled drifters are shown in Table 1 for the period of 1 month from 1st to 30th June. Drifter \#6 leaves the model domain very early in all model runs and is not shown in the table.

The most significant changes in travel distance are seen for the drifters released either inside the farm (\#7) or relatively close to it (\#8; deployed $24 \mathrm{~km}$ from the centre). Movements of the drifter \#8 are influenced by the local intensification of currents next to the farm due to effect of current "avoiding" the farm.

Similar to the wind farms, the energy extraction efficiency is dependent on the layout of the farm. An example show- 

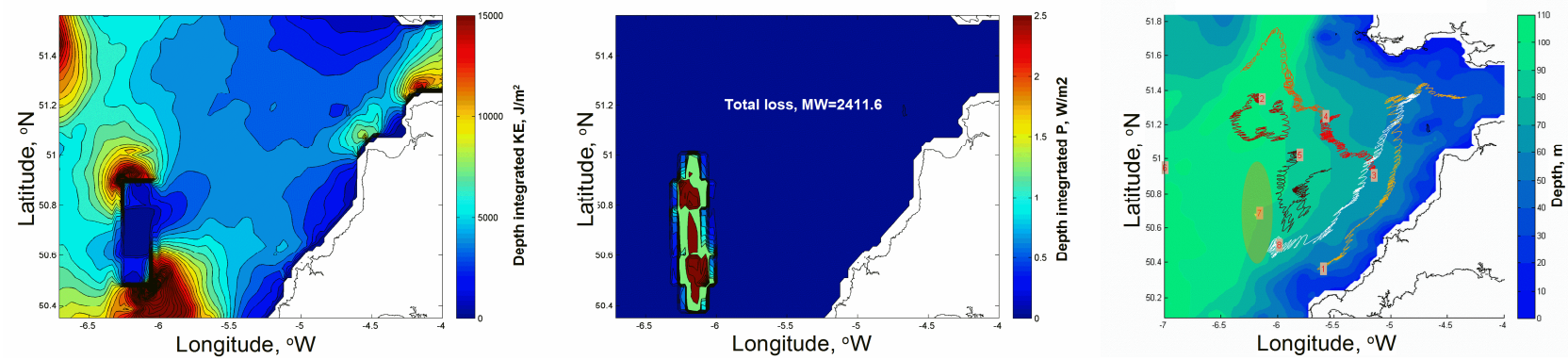

Fig. 8. Effect of the farm shape on the main parameters of the flow. Left panel- kinetic energy per unit area $\left(\mathrm{J} \mathrm{m}^{-2}\right)$; middle panel- mean extracted power in $\mathrm{W} \mathrm{m}^{-2}$; right panel - simulated trajectories of Lagrangean drifters drogued at $22.5 \mathrm{~m}$ depth, for a farm extended along the meridian. The rated capacity of the farm (i.e. the number of turbines) is the same as for high-rated circular farm shown in Figs. 5-7. The shape of the farm is shown by a shaded oval on the right panel.

ing variations of the main parameters of the flow and the level of extracted energy for a farm of elongated shape is shown in Fig. 8. The farm in Fig. 8 has the same value of $\int \alpha d x d y d z$, i.e. the same number of turbines as the high energy farm shown in Fig. 5c. We can see that the shape of the tidal farm affects both the values of extracted energy and the impact on the environment; however the detailed study of this issue is a topic for future research.

The combined analysis of changes in the horizontal distribution of kinetic energy and Lagrangean drifters shows that in addition to a local effect (within the area of the farm) there is a regional impact, in particular on residual currents. This impact is similar to the effect of wave energy extraction on wave heights, which was shown to be felt as far as $30 \mathrm{~km}$ from the wave energy "farm" (Millar et al., 2006). Neill et al. (2009) used a 1-D sectionally-averaged model to show that energy extraction at discrete locations can alter sediment transport in the Bristol Channel well away from the turbine, about $120 \mathrm{~km}$. It is likely that collective action of the turbines on the open shelf could also alter sediment transport well outside the farm, however this subject needs further investigation using a 3-D approach. In contrast to the common opinion that "... because the tidal flow continues unchecked, these technologies (i.e. submerged turbines) have a minimal environmental impact" (The renewable energy centre, 2010), our results show that there is a region-wide effect on residual currents in the shelf sea, and a careful scientific analysis should be done to assess whether this effect could be harmful. This study shows that both energy extraction estimates and effects on region wide circulation depend on a complex combination of factors, and the specific figures given in the paper should be generally considered as first estimates.

\section{Conclusions}

Numerical simulation of the impact of a hypothetical tidal stream energy farm on the currents in a shelf sea has shown that at relatively high levels of rated power generation capacity the farm can introduce noticeable changes to the speeds and the circulation patterns as far as a hundred kilometres away from the farm. Due to the extraction of energy from the ocean currents, the flow of water slows down. This effect causes the maximum extractable power to be significantly less than the estimates of the resource calculated using undisturbed, naturally flowing currents. In the case study $(\mathrm{H})$ presented here, the maximum extractable energy is at least 14 times lower than if the currents had not been disturbed by the farm. The effect of the reduction of extractable power in the open shelf sea is much stronger than it is in a channel.

The impact on the instantaneous current speed and the kinetic energy contained in the movement of water by a circular farm of $12 \mathrm{~km}$ in diameter is felt mostly within the farm and at a close range of about $10-20 \mathrm{~km}$. However residual, tidally averaged currents are modified as far as a hundred kilometres away, and the pathways of passive tracers, which are modelled with Lagrangean floats and mimic the dispersion of pollution, are disturbed.

It should be noted that the purpose of this study was to give only the first estimates of the reduction in resource and the region-wide impacts of the energy farm located in the open shelf sea. As such the numerical values given in this paper should be considered purely as examples. Both effects: the reduction of extractable energy and environmental impact could be lower or larger depending on the shape, size and location of the farm. The change in residual currents could also vary with season and in different years as these currents incorporate weather-dependent wind-driven and seasonal density-driven components in addition to the rectified tidal flow. 
Acknowledgements. The author is grateful to D. Aleynik for his help in setting up the POLCOMS model for the baseline study, to S. Wakelin for providing tidal boundary conditions and to the PRIMaRE colleagues for encouraging discussions. The post-processing methodology used in this paper was developed with support from EU FP6 SESAME and EU FP7 MyOcean projects.

Edited by: J. M. Huthnance

\section{References}

Bjorn, S., Duan, J., McWilliams, J. C., Münnich, M., and Neelin, J. D.: Entrainment, Rayleigh Friction, and Boundary Layer Winds over the Tropical Pacific, J. Climate, 15, 30-44, 2002.

Black and Veatch Consulting Ltd.: Phase I UK tidal stream energy resource assessment, Technical report 107799/D/2200/03, Carbon Trust, London, July 2005.

Boyer, T. P., Levitus, S., Garcia, H., Locarnini, R. A., Stephens, C., and Antonov, J.: Objective analyses of annual, seasonal, and monthly temperature and salinity for the world ocean on a $0.25^{\circ}$ grid, Int. J. Climatol., 25, 931-945, doi:10.1002/JOC.1173, 2005.

Bryden, I. G. and Couch, S. J.: Marine energy extraction: tidal resource analysis, J. Renew. Energy, 31(2), 133-139, 2006.

Carballo, R., Iglesias, G., and Castro, A.: Numerical model evaluation of tidal stream energy resources in the Ria de Muros (NW Spain), Renew. Energy, 34, 1517-1524, 2009.

DE: Department of Energy (DE), Central Electricity Generating Board and Severn Tidal Power Group, Severn Barrage Project: Detailed Report (EP57), HMSO, London, 99 pp., 1989.

DTI: Our energy future - creating a low carbon economy, Department of Trade and Industry white paper, 142 pp., 2003.

Egbert, G. D. and Ray, R. D.: Significant dissipation of tidal energy in the deep ocean inferred from satellite altimeter data, Nature, 405, 775-778, 2000.

ETOPO2: ETOPO2v2 Global Gridded 2-minute Database, NOAA, available at: http://www.ngdc.noaa.gov/mgg/global/etopo2.html (last access: 23 September 2010), 2006.

Frandsen, S. T.: Turbulence and turbulence-generated structural loading in wind turbine clusters, Ris $\varnothing$ National Laboratory Report Nr. Ris $\varnothing-\mathrm{R}-1188(\mathrm{EN})$, available at: http://130.226.56.153/ rispubl/VEA/veapdf/ris-r-1188.pdf (last access: 23 December 2010), January 2007.

Frohboese, P. and Schmuck, C.: Thrust coefficients used for estimation of wake effects for fatigue load calculation, European Wind Energy Conference, Warsaw, Poland; available at: http://www.gl-garradhassan.com/assets/downloads/ EWEC2010_PAPER_CT-Curves_Frohboese_Final.pdf, (last access: 18 December 2010), 2010.

Garrett, C. and Cummins, P.: Limits to tidal current power, Renew. Energy, 33, 2485-2490, 2008.

Gorlov, A. M.: Tidal Energy, in: Encyclopedia of Ocean Sciences, Academic Press, Oxford, 2955-2960, 2001.

Hansen, M. O. L.: Aerodynamics of Wind Turbines, Earthscan Publications, 190 pp., 2008.
Holt, J. T., Allen, J. I., Proctor, R., and Gilbert, F.: Error quantification of a high resolution coupled hydrodynamic-ecosystem coastal-ocean model: part 1 model overview and assessment of the hydrodynamics, J. Mar. Sys., 57, 167-188, 2005.

Holt, J. T. and James, I. D.: An s-coordinate density evolving model of the northwest European continental shelf Part 1 model description and density structure, J. Geophys. Res., 106, 14015-14034, 2001.

Huntley, D. A.: Tides on the North-West European Continental shelf, in: North-west European Shelf Seas: Physical and Chemical Oceanography and Physical Resources v. 2: Sea Bed and the Sea in Motion, edited by: Banner F. T., Elsevier oceanography series, 301-352, 1980.

IEA: Key World energy statistics, available at: http://www.iea. org/textbase/nppdf/free/2009/key_stats_2009.pdf (last access: 14 September 2010), International Energy Agency, 2009.

Leishman, J. G.: Principles of helicopter aerodynamics, Cambridge University Press, 496 pp., 2000.

Lewis, K., Allen, J. I., Richardson, A. J., and Holt, J. T.: Error quantification of a high resolution coupled hydrodynamic-ecosystem coastal-ocean model: Part3, validation with Continuous Plankton Recorder data, J. Mar. Sys., 63, 209-224, 2006.

Li, Y., Lence, B. J., and Calisal, S. M.: Modeling the Energy Output from an In-Stream Tidal Turbine Farm, J. Computers, 4(4), 288294, 2009.

List of largest power stations in the world: available at: http://en. wikipedia.org/wiki/List_of_largest_power_stations_in_the_world (last access: 14 September 2010), Wikipedia, 2010.

Marine Current turbines, available at: http://www.marineturbines. com/21/, last access: 14 September 2010.

Millar, D. L., Smith, H. C. M., and Reeve, D. E.: Modelling Studies of the Sensitivity of the Shoreline Wave Climate to the Proposed Wave Farm Development off the North Coast of Cornwall, Ocean Engineer., 34(5-6), 884-901, 2006.

Mueller, M. and Wallace, R.: Enabling science and technology for marine renewable energy, Energy Policy, 36, 4376-4382, 2008.

Munk, W. and Wunsch, C.: Abyssal recipes II: energetics of tidal and wind mixing, Deep Sea Res. Part I, 45(12), 1977-2010, 1998.

Neill, S. P., Litt, E. J., Couch, S. J., and Davies, A. G.: The impact of tidal stream turbines on large-scale sediment dynamics, Renew. Energy, 34, 2803-2812, 2009.

Pedlosky, J.: Geophysical Fluid Dynamics, Springer, 728 pp., 1990. SDC: Sustainable Development Commission (SDC) Tidal Power in the UK: Research Report 3 - Severn Barrage Proposals, 2007, available at: http://www.sd-commission.org.uk/publications/ downloads/TidalPowerUK3-Severn_barrage_proposals.pdf (last access: 21 February 2011), 2007.

Shapiro, G. I., Huntley, D. A., and Aleynik, D. L.: Circulation and sediment transport patterns in eastern Celtic Sea, 14th Biennial Challenger Conference for Marine Science, National Oceanography Centre, Southampton, 6-9 September 2010, available at: www.challenger2010.org.uk (last access: 15 September 2010), 2010.

Siddorn, J. R., Allen, J. I., Blackford, J. C., Gilbert, F. J., Holt, J. T., Holt, M. W., Osborne, J. P., Proctor, R., and Mills, D. K.: Modelling the hydrodynamics and ecosystem of the North-West European continental shelf for operational oceanography, J. Mar. Sys., 65, 417-429, 2007. 
Soerensen, H. C. and Weinstein, A.: Ocean Energy: Position paper for IPCC. Key Note Paper for the IPCC Scoping Conference on Renewable Energy, Lübeck, Germany, available at: http://www.eu-oea.com/euoea/files/ccLibraryFiles/Filename/ 000000000400/Ocean_Energy_IPCC_final.pdf (last access: 14 September 2010), January 2008.

Stiesdal, H., Petersen, K. O., and Thisted J.: An adaptable 1MW wind turbine, in: Proceedings of the European Wind Energy Conference, Nice, France 1-5 March 1999, edited by: Petersen, E. L., James \& James Science Publishers, 741-744, 1999.

The renewable energy centre, available at: http://www. therenewableenergycentre.co.uk/contactus.html, last access: 15 September 2010.
Xia, J., Falconer, R. A., and Lin, B.: Impact of different tidal renewable energy projects on the hydrodynamic processes in the Severn Estuary, UK, Ocean Modelling, 32, 86-104, 2010.

Walkington, I. and Burrows, R.: Modelling tidal stream power potential, Appl. Ocean Res., 31, 239-245, 2009.

Williams, G. E.: Geological constraints on the Precambrian history of Earth's rotation and the Moon's orbit, Rev. Geophys., 38(1), 37-59, 2000. 